PROCEEDINGS OF THE

AMERICAN MATHEMATICAL SOCIETY

Volume 132, Number 9, Pages 2529-2535

S 0002-9939(04)07502-1

Article electronically published on April 21, 2004

\title{
ESSENTIAL DOMAINS AND TWO CONJECTURES IN DIMENSION THEORY
}

\author{
M. FONTANA AND S. KABBAJ
}

(Communicated by Bernd Ulrich)

\begin{abstract}
This note investigates two long-standing conjectures on the Krull dimension of integer-valued polynomial rings and of polynomial rings in the context of (locally) essential domains.
\end{abstract}

\section{INTRODUCTION}

Let $R$ be an integral domain with quotient field $K$, and let $\operatorname{Int}(R):=\{f \in$ $K[X]: f(R) \subseteq R\}$ be the ring of integer-valued polynomials over $R$. Considerable work, part of it summarized by Cahen and Chabert 7], has been concerned with various aspects of integer-valued polynomial rings. A central question concerning $\operatorname{Int}(R)$ is to describe its prime spectrum and, hence, to evaluate its Krull dimension. Several authors tackled this problem, and many satisfactory results were obtained for various classes of rings such as Dedekind domains [9, 10], Noetherian domains 10], valuation and pseudo-valuation domains [8, and pseudo-valuation domains of type $n$ 33. A well-known feature is that $\operatorname{dim}(R[X])-1 \leq \operatorname{dim}(\operatorname{Int}(R))$ for any integral domain $R[5]$. However, the problem of improving the upper bound $\operatorname{dim}(\operatorname{Int}(R)) \leq \operatorname{dim}_{v}(R[X])=\operatorname{dim}_{v}(R)+1$ [16], where $\operatorname{dim}_{v}(R)$ denotes the valuative dimension of $R$, is still elusively open in general. This is due, in part, to the fact that the fiber in $\operatorname{Int}(R)$ of a maximal ideal of $R$ may have any dimension [5, Example 4.3] (this stands for the main difference between polynomial rings and integer-valued polynomial rings). It is noteworthy that all examples conceived in the literature for testing $\operatorname{dim}(\operatorname{Int}(R))$ satisfy the inequality $\operatorname{dim}(\operatorname{Int}(R)) \leq \operatorname{dim}(R[X])$. In [16, 17, we undertook an extensive study, using two different approaches, in order to grasp this phenomenon. We then obtained further evidence for the validity of the conjecture

$$
\operatorname{dim}(\operatorname{Int}(R)) \leq \operatorname{dim}(R[X]) \text { for any integral domain } R .
$$

Received by the editors January 15, 2003.

2000 Mathematics Subject Classification. Primary 13C15, 13F20, 13F05, 13G05, 13B02, $13 \mathrm{~B} 30$.

Key words and phrases. Krull dimension, valuative dimension, Jaffard domain, integer-valued polynomial ring, essential domain, Krull domain, UFD, PVMD, Kronecker function ring, star operation.

The first author was partially supported by a research grant MIUR 2001/2002 (Cofin 2000MM01192794). The second author was supported by the Arab Fund for Economic and Social Development.

This work was done while both authors were visiting Harvard University. 
The current situation can be described as follows. $\left(\mathcal{C}_{1}\right)$ turned out to be true in three large (presumably different) classes of commutative rings, namely, (a) Krulltype domains, e.g., unique factorization domains (UFDs) or Krull domains [22, 17]; (b) pseudo-valuation domains of type $n$ [17]; and (c) Jaffard domains [16, 5].

A finite-dimensional domain $R$ is said to be Jaffard if $\operatorname{dim}\left(R\left[X_{1}, \ldots, X_{n}\right]\right)=$ $n+\operatorname{dim}(R)$ for all $n \geq 1$; equivalently, if $\operatorname{dim}(R)=\operatorname{dim}_{v}(R)$ [1, 4, 14 19] 27. The class of Jaffard domains contains most of the well-known classes of finitedimensional rings involved in dimension theory of commutative rings, such as Noetherian domains 29], Prüfer domains [19], universally catenarian domains [3], and stably strong $S$-domains 28 30. However, the question of establishing or denying a possible connection to the family of Krull-like domains is still unsolved. In this vein, Bouvier's conjecture (initially announced during a 1985 graduate course at the University of Lyon I) states that

finite-dimensional Krull domains, or more particularly UFDs, need not be Jaffard domains.

Since the Krull property is stable under adjunction of indeterminates, the problem merely deflates to the existence of a Krull domain $R$ with $1+\operatorname{dim}(R) \supsetneqq \operatorname{dim}(R[X])$. It is notable that the rare non-Noetherian finite-dimensional UFDs or Krull domains existing in the literature do defeat $\left(\mathcal{C}_{2}\right)$, since all are Jaffard [2, 12, 13, 14, 18, 20. So do the examples of non-Prüfer finite-dimensional Prüfer $v$-multiplication domains (PVMDs) [21, 26, 31, 34]; as a matter of fact, these mainly arise as polynomial rings over Prüfer domains or as pullbacks, and both settings either yield Jaffard domains or turn out to be inconclusive (in terms of allowing the construction of counterexamples) [1 [15]. In order to find the missing link, one has then to dig beyond the context of PVMDs.

Essential domains happen to offer such a suitable context for $\left(\mathcal{C}_{2}\right)$ as well as a common environment for both conjectures $\left(\mathcal{C}_{1}\right)$ and $\left(\mathcal{C}_{2}\right)$, though these have developed in two dissimilar milieus. An integral domain $R$ is said to be essential if $R$ is an intersection of valuation rings that are localizations of $R[23$. Since this notion does not carry up to localizations, $R$ is said to be locally essential if $R_{p}$ is essential for each $p \in \operatorname{Spec}(R)$. Notice that the locally essential domains correspond to the $P$-domains in the sense of Mott and Zafrullah 31. PVMDs and almost Krull domains [19, p. 538] are perhaps the most important examples of locally essential domains. Recall that Heinzer constructed in 24 an example of an essential domain that is not locally essential. Also, it is worth noticing that Heinzer and Ohm's example 25. of an essential domain that is not a PVMD is, in fact, locally essential (cf. [31, Example 2.1]). Finally, recall that a Krull-type domain is a PVMD in which no nonzero element belongs to an infinite number of maximal $t$-ideals 21 . We have thus the implications shown in the diagram within the family of Krull-like domains:

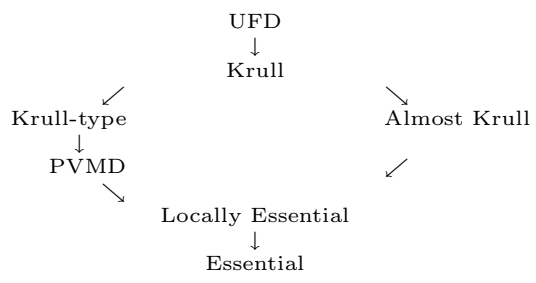


The purpose of this note is twofold. First, we state a result that widens the domain of validity of $\left(\mathcal{C}_{1}\right)$ to the class of locally essential domains. It is well known that $\left(\mathcal{C}_{1}\right)$ holds for Jaffard domains too [16, [5]. So one may enlarge the scope of study of $\left(\mathcal{C}_{2}\right)$, discussed above, and legitimately raise the following problem:

$\left(\mathcal{C}_{2}^{\prime}\right) \quad$ Is every finite-dimensional (locally) essential domain Jaffard?

Clearly, an affirmative answer to $\left(\mathcal{C}_{2}^{\prime}\right)$ will definitely defeat $\left(\mathcal{C}_{2}\right)$, while a negative answer will partially resolve $\left(\mathcal{C}_{2}\right)$ for the class of (locally) essential domains. Our second aim is to show that the rare constructions of nontrivial (locally) essential domains (i.e., non-PVMD) existing in the literature yield Jaffard domains, making $\left(\mathcal{C}^{\prime}{ }_{2}\right)$ an open problem. Consequently, a settlement of $\left(\mathcal{C}_{2}\right)$ seems out of reach at present.

\section{Result And EXAMPLE}

In the first part of this section, we establish the following result.

Theorem 2.1. For any locally essential domain $R, \operatorname{dim}(\operatorname{Int}(R))=\operatorname{dim}(R[X])$.

Proof. Assume that $R$ is finite-dimensional and $R \neq K$, where $K$ denotes the quotient field of $R$. Let $R=\bigcap_{p \in \Delta} R_{p}$ be a locally essential domain, where $\Delta \subseteq$ $\operatorname{Spec}(R)$. Set

$$
\begin{aligned}
& \Delta_{1}:=\left\{p \in \Delta: R_{p} \text { is a DVR }\right\} \\
& \Delta_{2}:=\left\{p \in \Delta: R_{p} \text { is a valuation domain but not a DVR }\right\}
\end{aligned}
$$

We wish to show first that $\operatorname{dim}(\operatorname{Int}(R)) \leq \operatorname{dim}(R[X])$. Let $M$ be a maximal ideal of $\operatorname{Int}(R)$ such that $\operatorname{dim}(\operatorname{Int}(R))=\operatorname{ht}(M)$ and let $\mathcal{M}:=M \cap R$. Without loss of generality, we may assume that $\mathcal{M}$ is maximal in $R$ with a finite residue field. We always have $R_{\mathcal{M}}[X] \subseteq(\operatorname{Int}(R))_{\mathcal{M}} \subseteq \operatorname{Int}\left(R_{\mathcal{M}}\right)$ 6. Corollaires (4), p. 303]. If $\mathcal{M} \in \Delta_{1}$, then $R_{\mathcal{M}}[X]$ is a two-dimensional Jaffard domain ([32, Theorem 4] and [1. Proposition 1.2]). So the inclusion $R_{\mathcal{M}}[X] \subseteq(\operatorname{Int}(R))_{\mathcal{M}}$ yields $\operatorname{dim}\left(\left((\operatorname{Int}(R))_{\mathcal{M}}\right) \leq\right.$ $\operatorname{dim}_{v}\left(R_{\mathcal{M}}[X]\right)=\operatorname{dim}\left(R_{\mathcal{M}}[X]\right)$. Thus, $\operatorname{dim}\left(\left((\operatorname{Int}(R))_{\mathcal{M}}\right)=\operatorname{dim}\left(R_{\mathcal{M}}[X]\right)=2\right.$. If $\mathcal{M} \in \Delta_{2}$, then $\operatorname{Int}\left(R_{\mathcal{M}}\right)=R_{\mathcal{M}}[X]=(\operatorname{Int}(R))_{\mathcal{M}}$ [6] Exemples (5), p. 302]. If $\mathcal{M} \notin \Delta$, then $R_{\mathcal{M}}=\bigcap_{p \in \Delta, p \subsetneq \mathcal{M}} R_{p}$, since $R$ is a locally essential domain. So, by 6. Corollaires (3), p. 303], $\operatorname{Int}\left(R_{\mathcal{M}}\right)=\bigcap_{p \in \Delta, p \subsetneq \mathcal{M}} \operatorname{Int}\left(R_{p}\right)=\bigcap_{p \in \Delta, p \subsetneq \mathcal{M}} R_{p}[X]=$ $R_{\mathcal{M}}[X]=(\operatorname{Int}(R))_{\mathcal{M}}$. In all cases, we have $\operatorname{dim}(\operatorname{Int}(R))=\operatorname{dim}\left(\left((\operatorname{Int}(R))_{\mathcal{M}}\right)=\right.$ $\operatorname{dim}\left(R_{\mathcal{M}}[X]\right) \leq \operatorname{dim}(R[X])$, as desired.

We now establish the inverse inequality $\operatorname{dim}(R[X]) \leq \operatorname{dim}(\operatorname{Int}(R))$. Let $M$ be a maximal ideal of $R[X]$ such that $\operatorname{dim}(R[X])=\operatorname{ht}(M)$, and $\mathcal{M}:=M \cap R$. Necessarily, $\mathcal{M}$ is maximal in $R$. Furthermore, we may assume that $\mathcal{M}$ has a finite residue field. If $\mathcal{M} \in \Delta_{2}$ or $\mathcal{M} \notin \Delta$, similar arguments as above lead to $R_{\mathcal{M}}[X]=(\operatorname{Int}(R))_{\mathcal{M}}$ and hence to the conclusion. Next, suppose $\mathcal{M} \in \Delta_{1}$. Then, $\operatorname{Int}\left(R_{\mathcal{M}}\right)$ is a two-dimensional (Prüfer) domain [11. Let $(0) \varsubsetneqq P_{1} \varsubsetneqq P_{2}$ be a maximal chain of prime ideals in $\operatorname{Spec}\left(\operatorname{Int}\left(R_{\mathcal{M}}\right)\right)$. Clearly, it contracts to $(0) \varsubsetneqq \mathcal{M} R_{\mathcal{M}}$ in $\operatorname{Spec}\left(R_{\mathcal{M}}\right)$. Furthermore, by [5, Corollaire 5.4], $P_{1}$ contracts to (0). Therefore, by [10] Proposition 1.3], $P_{1}=f K[X] \cap \operatorname{Int}\left(R_{\mathcal{M}}\right)$, for some irreducible polynomial $f \in K[X]$. This yields in $\operatorname{Spec}\left(R_{\mathcal{M}}[X]\right)$ the maximal chain

$$
\text { (0) } \varsubsetneqq P_{1} \cap R_{\mathcal{M}}[X]=f K[X] \cap R_{\mathcal{M}}[X] \varsubsetneqq P_{2} \cap R_{\mathcal{M}}[X]
$$


which induces in $\operatorname{Spec}\left((\operatorname{Int}(R))_{\mathcal{M}}\right)$ the following maximal chain:

$$
(0) \varsubsetneqq P_{1} \cap(\operatorname{Int}(R))_{\mathcal{M}}=f K[X] \cap(\operatorname{Int}(R))_{\mathcal{M}} \varsubsetneqq P_{2} \cap(\operatorname{Int}(R))_{\mathcal{M}} .
$$

Consequently, in all cases, we obtain $\operatorname{dim}(R[X])=\operatorname{dim}\left(R_{\mathcal{M}}[X]\right)=\operatorname{dim}\left(\left((\operatorname{Int}(R))_{\mathcal{M}}\right)\right.$ $\leq \operatorname{dim}(\operatorname{Int}(R))$, to complete the proof of the theorem.

From [25, Proposition 1.8] and [19, Exercise 11, p. 539] we obtain the following.

Corollary 2.2. Let $R$ be a $P V M D$ or an almost Krull domain. Then $\operatorname{dim}(\operatorname{Int}(R))=$ $\operatorname{dim}(R[X])$.

In the second part of this section, we test the problem $\left(\mathcal{C}^{\prime}{ }_{2}\right)$, set up and discussed in the introduction, for the class of non-PVMD (locally) essential domains. These occur exclusively in the Heinzer-Ohm example [25] and Heinzer's example [24, both mentioned above. The first of them is a 2-dimensional Jaffard domain [31, Example 2.1]. Heinzer's example 24, too, is a 2-dimensional Jaffard domain by 14, Theorem 2.3]. Our next example shows that an enlargement of the scope of this construction still generates a large family of essential domains with nonessential localizations of arbitrary dimensions $\geq 2$, but unfortunately they are Jaffard domains.

Example 2.3. For any integer $r \geq 2$, there exists an $r$-dimensional essential Jaffard domain $D$ that is not locally essential.

Notice first that the case $r=2$ corresponds to Heinzer's example mentioned above. In order to increase the dimension, we modify Heinzer's original setting by considering Kronecker function rings via the $b$-operation. For the sake of completeness, we give below the details of this construction.

Let $R$ be an integral domain, $K$ its quotient field, $n$ a positive integer (or $n=\infty$ ), and $X, X_{1}, \ldots, X_{n}$ indeterminates over $K$. The $b$-operation on $R$ is the a.b. star operation defined by $I^{b}:=\bigcap\{W: W$ is a valuation overring of $R\}$, for every fractional ideal $I$ of $R$. Throughout, we shall use $\operatorname{Kr}_{K(X)}(R, b)$ to denote the Kronecker function ring of $R$ defined in $K(X)$ with respect to the $b$-operation on $R$, and $R\left(X_{1}, \ldots, X_{n}\right)$ to denote the Nagata ring associated to the polynomial ring $R\left[X_{1}, \ldots, X_{n}\right]$, obtained by localizing the latter with respect to the multiplicative system consisting of polynomials whose coefficients generate $R$.

Let $r$ be an integer $\geq 2$. Let $k_{0}$ be a field, and let $\left\{X_{n}: n \geq 1\right\}, Y,\left\{Z_{1}, \ldots, Z_{r-1}\right\}$ be indeterminates over $k_{0}$. Let $n$ be a positive integer. Set:

$$
\begin{array}{ll}
k_{n}:=k_{0}\left(X_{1}, \ldots, X_{n}\right), & k:=\bigcup_{n \geq 1} k_{n}, \\
F_{n}:=k_{n}\left(Z_{1}, \ldots, Z_{r-1}\right), & F:=\bigcup_{n \geq 1} F_{n}=k\left(Z_{1}, \ldots, Z_{r-1}\right), \\
K_{n}:=F_{n}(Y), & K:=\bigcup_{n \geq 1} K_{n}=F(Y), \\
M_{n}:=Y F_{n}[Y]_{(Y)}, & M:=\bigcup_{n \geq 1} M_{n}=Y F[Y]_{(Y)}, \\
A_{n}:=k_{n}+M_{n}, & A:=\bigcup_{n \geq 1} A_{n}=k+M, \\
V_{n}:=F_{n}[Y]_{(Y)}, & V:=\bigcup_{n \geq 1} V_{n}=F[Y]_{(Y)} .
\end{array}
$$

Note that, for each $n \geq 1, V$ and $V_{n}$ (resp., $A$ and $A_{n}$ ) are one-dimensional discrete valuation domains (resp., pseudo-valuation domains) and $\operatorname{dim}_{v}(A)=$ $\operatorname{dim}_{v}\left(A_{n}\right)=r$. For each $n \geq 1$, set $X_{n}^{\prime}:=\frac{1+Y X_{n}}{Y}$. Clearly, $K_{n}=K_{n-1}\left(X_{n}\right)=$ $K_{n-1}\left(X_{n}^{\prime}\right)$. Next, we define inductively two sequences of integral domains $\left(B_{n}\right)_{n \geq 2}$ 
and $\left(D_{n}\right)_{n \geq 1}$ as follows:

$$
\begin{array}{ll} 
& D_{1}:=A_{1}, \\
B_{2}:=\operatorname{Kr}_{K_{1}\left(X_{2}^{\prime}\right)}\left(D_{1}, b\right), & D_{2}:=B_{2} \cap A_{2}, \\
B_{n}:=\operatorname{Kr}_{K_{n-1}\left(X_{n}^{\prime}\right)}\left(D_{n-1}, b\right), & D_{n}:=B_{n} \cap A_{n}, \text { for } n \geq 3 .
\end{array}
$$

For $n \geq 2$, let $\mathcal{M}_{n}:=M_{n} \cap D_{n}\left(\subset D_{n}=B_{n} \cap A_{n} \subseteq A_{n}\right)$, where $M_{n}$ is the maximal ideal of $A_{n}$.

Claim 2.4. (1) $B_{n}$ is an $r$-dimensional Bézout domain.

(2) $B_{n} \cap K_{n-1}=D_{n-1} \subseteq A_{n-1}=A_{n} \cap K_{n-1}$.

(3) $D_{n} \cap K_{n-1}=D_{n-1}$.

(4) $D_{n}\left[X_{n}^{\prime}\right]=B_{n}$ and $\left(D_{n}\right)_{\mathcal{M}_{n}}=D_{n}\left[\frac{1}{Y X_{n}^{\prime}}\right]=A_{n}$, with $\frac{1}{X_{n}^{\prime}}$ and $Y X_{n}^{\prime} \in D_{n}$.

(5) $\mathcal{M}_{n}$ is a height-one maximal ideal of $D_{n}$ with $\mathcal{M}_{n} \cap K_{n-1}=\mathcal{M}_{n-1}$.

(6) For each $q \in \operatorname{Spec}\left(D_{n}\right)$ with $q \neq \mathcal{M}_{n}$ there exists a unique prime ideal $Q \in \operatorname{Spec}\left(B_{n}\right)$ contracting to $q$ in $D_{n}$ with the property that $\left(D_{n}\right)_{q}=\left(B_{n}\right)_{Q}$.

(7) $B_{n}=\bigcap\left\{\left(D_{n}\right)_{q}: q \in \operatorname{Spec}\left(D_{n}\right)\right.$ and $\left.q \neq \mathcal{M}_{n}\right\}$.

(8) For each $q^{\prime} \in \operatorname{Spec}\left(D_{n-1}\right)$ with $q^{\prime} \neq \mathcal{M}_{n-1}$ there exists a unique prime ideal $q\left(\neq \mathcal{M}_{n}\right) \in \operatorname{Spec}\left(D_{n}\right)$ contracting to $q^{\prime}$ in $D_{n-1}$ such that $\left(D_{n}\right)_{q}=\left(D_{n-1}\right)_{q^{\prime}}\left(X_{n}^{\prime}\right)$.

Proof. Similar arguments as in 24] lead to (1)-(7).

(8) By $(7), B_{n-1} \subseteq\left(D_{n-1}\right)_{q^{\prime}}$, and hence $\left(D_{n-1}\right)_{q^{\prime}}$ is a valuation domain in $K_{n-1}$ of dimension $\leq r$ containing $D_{n-1}$. Since $B_{n}$ is the Kronecker function ring of $D_{n-1}$ defined in $K_{n-1}\left(X_{n}^{\prime}\right)$ by all valuation overrings of $D_{n-1}$, then $\left(D_{n-1}\right)_{q^{\prime}}$ has a unique extension in $K_{n-1}\left(X_{n}^{\prime}\right)$, that is a valuation overring of $B_{n}$, that is, $\left(D_{n-1}\right)_{q^{\prime}}\left(X_{n}^{\prime}\right)$. By (7), the center $q$ of $\left(D_{n-1}\right)_{q^{\prime}}\left(X_{n}^{\prime}\right)$ in $D_{n}$ is the unique prime ideal of $D_{n}$ lying over $q^{\prime}$ with the property that $\left(D_{n}\right)_{q}=\left(D_{n-1}\right)_{q^{\prime}}\left(X_{n}^{\prime}\right)$.

Set $D:=\bigcup_{n \geq 1} D_{n}$ and $\mathcal{M}:=\bigcup_{n \geq 2} \mathcal{M}_{n}$. It is obvious that $D \subseteq A=\bigcup_{n \geq 1} A_{n}$. Claim 2.5. $D_{\mathcal{M}}=A$, and $\mathcal{M}$ is a height-one maximal ideal in $D$.

Proof. This is an easy consequence of Claim 2.4(4), since $\mathcal{M}_{n}=\mathcal{M} \cap D_{n}$ for each $n$.

Let $q \in \operatorname{Spec}(D)$ with $q \neq \mathcal{M}$. Then, for some $m \geq 2$, we have in $D_{m}, q_{m}:=$ $q \cap D_{m} \neq \mathcal{M}_{m}=\mathcal{M} \cap D_{m}$. So, by Claim 2.4(6), $B_{m} \subseteq\left(D_{m}\right)_{q_{m}}$. Hence $\left(D_{m}\right)_{q_{m}}$ is a valuation overring of $B_{m}$ of dimension $\leq r$, whence, by Claim $2.4(8), D_{q}=$ $\bigcup_{n \geq 1}\left(D_{n}\right)_{q_{n}}=\left(D_{m}\right)_{q_{m}}\left(X_{m+1}^{\prime}, \ldots\right)$ is still a valuation domain of dimension $\leq r$.

Claim 2.6. $D=\bigcap\left\{D_{q}: q \in \operatorname{Spec}(D)\right.$ and $\left.q \neq \mathcal{M}\right\}$.

Proof. Similar to [24].

From Claims 2.5 and 2.6 we obtain:

Conclusion 2.7. $D$ is an essential domain with a nonessential localization, and $\operatorname{dim}(D)=\operatorname{dim}_{v}(D)=r$.

\section{REFERENCES}

[1] D. F. Anderson, A. Bouvier, D. E. Dobbs, M. Fontana, and S. Kabbaj, On Jaffard domains, Expo. Math., 6 (2) (1988), 145-175. MR 89c:13014

[2] D. F. Anderson and S. B. Mulay, Noncatenary factorial domains, Comm. Algebra, 17 (1989), 1179-1185. MR 90c:13021 
[3] A. Bouvier, D. E. Dobbs, and M. Fontana, Universally catenarian integral domains, Advances in Math., 72 (1988), 211-238. MR 89m:13007

[4] A. Bouvier and S. Kabbaj, Examples of Jaffard domains, J. Pure Appl. Algebra, 54 (1988), 155-165. MR 89k:13019

[5] P.-J. Cahen, Dimension de l'anneau des polynômes à valeurs entières, Manuscripta Math., 67 (1990), 333-343. MR 91e:13010

[6] P.-J. Cahen and J.-L. Chabert, Coefficients et valeurs d'un polynôme, Bull. Sci. Math. France, 95 (1971), 295-304. MR 45:5126

[7] P.-J. Cahen and J.-L. Chabert, Integer-Valued Polynomials, Amer. Math. Soc. Surveys and Monographs 48, 1997. MR 98a:13002

[8] P.-J. Cahen and Y. Haouat, Polynômes à valeurs entières sur un anneau de pseudovaluation, Manuscripta Math., 61 (1988), 23-31. MR 89f:13007

[9] J.-L. Chabert, Anneaux de polynômes à valeurs entières et anneaux de Fatou, Bull. Soc. Math. France, 99 (1971), 273-283. MR 46:1780

[10] J.-L. Chabert, Les idéaux premiers de l'anneau des polynômes à valeurs entières, J. Reine Angew. Math., 293/294 (1977), 275-283. MR 56:345

[11] J.-L. Chabert, Un anneau de Prüfer, J. Algebra, 107 (1987), 1-16. MR 88i:13022

[12] J. David, A non-Noetherian factorial ring, Trans. Amer. Math. Soc., 169 (1972), 495-502. MR 46:7229

[13] J. David, A characteristic zero non-Noetherian factorial ring of dimension three, Trans. Amer. Math. Soc., 180 (1973), 315-325. MR 48:3951

[14] D. E. Dobbs, M. Fontana, and S. Kabbaj, Direct limits of Jaffard domains and S-domains, Comment. Math. Univ. St. Paul., 39 (2) (1990), 143-155. MR 91m:13012

[15] M. Fontana and S. Gabelli, On the class group and the local class group of a pullback, J. Algebra, 181 (1996), 803-835. MR 97h:13011

[16] M. Fontana, L. Izelgue, S. Kabbaj, and F. Tartarone, On the Krull dimension of domains on integer-valued polynomials, Expo. Math., 15 (1997), 433-465. MR 98m:13027

[17] M. Fontana, L. Izelgue, S. Kabbaj, and F. Tartarone, Polynomial closure in essential domains and pullbacks, Lecture Notes in Pure and Appl. Math., Vol. 205, Dekker, New York, 1999, pp. 307-321. MR 2001g:13045

[18] K. Fujita, Three-dimensional unique factorization domain which is not catenary, J. Algebra 49 (1977), no. 2, 411-414. MR 57:6000

[19] R. Gilmer, Multiplicative Ideal Theory, Marcel Dekker, New York, 1972. MR 55:323

[20] R. Gilmer, A two-dimensional non-Noetherian factorial ring, Proc. Amer. Math. Soc. 44 (1974), 25-30. MR 49:281

[21] M. Griffin, Some results on v-multiplication rings, Canad. J. Math., 19 (1967), 710-722. MR 35:6665

[22] M. Griffin, Rings of Krull type, J. Reine Angew. Math., 229 (1968), 1-27. MR 36:3778

[23] M. Griffin, Families of finite character and essential valuations, Trans. Amer. Math. Soc., 130 (1968), 75-85. MR 36:1423

[24] W. Heinzer, An essential integral domain with a nonessential localization, Canad. J. Math., 33 (1981), 400-403. MR 82k:13006

[25] W. Heinzer and J. Ohm, An essential ring which is not a v-multiplication ring, Canad. J. Math., 25 (1973), 856-861. MR 48:6093

[26] E. Houston, S. B. Malik, and J. L. Mott, Characterizations of *-multiplication domains, Canad. Math. Bull., 27 (1984), 48-52. MR 85d:13026

[27] P. Jaffard, Théorie de la dimension dans les anneaux de polynômes, Mém. Sci. Math. 146, Gauthier-Villars, Paris, 1960. MR 22:8038

[28] S. Kabbaj, Sur les S-domaines forts de Kaplansky, J. Algebra 137 (1991), 400-415. MR 92d:13008

[29] I. Kaplansky, Commutative Rings, The University of Chicago Press, Chicago, 1974. MR 49:10674

[30] S. Malik and J. L. Mott, Strong S-domains, J. Pure Appl. Algebra, 28 (1983), 249-264. MR 84m:13017

[31] J. L. Mott and M. Zafrullah, On Prüfer v-multiplication domains, Manuscripta Math., 35 (1981), 1-26. MR 83d:13026

[32] A. Seidenberg, On the dimension theory of rings, II, Pacific J. Math., 4 (1954), 603-614. MR 16:441g 
[33] F. Tartarone, On the Krull dimension of $\operatorname{Int}(D)$ when $D$ is a pullback, Lecture Notes in Pure and Appl. Math., Dekker, 185 (1997), 457-476. MR 98b:13011

[34] M. Zafrullah, Some polynomial characterizations of Prüfer v-multiplication domains, J. Pure Appl. Algebra, 32 (1984), 231-237. MR 85j:13012

Dipartimento di Matematica, Università degli Studi "Roma Tre", Largo San L. MuriALDO 1, 00146 ROMA, ITALY

E-mail address: fontana@mat.uniroma3.it

Department of Mathematics, P.O. Box 5046, KFuPM, Dhahran 31261, Saudi Arabia

E-mail address: kabbaj@kfupm.edu.sa 